\title{
A Remarkable Case of Superior Mesenteric Artery Syndrome
}

\author{
Authors \\ Dr Subhasish Panda ${ }^{1}$, Dr S.Nisa ${ }^{2}$, Dr Savitri Bhagat ${ }^{3}$, Dr Braja B Panda ${ }^{4}$, \\ Dr Bararuchi Dash ${ }^{5}$, Dr Rajesh Pattanaik ${ }^{6}$, Dr Goguldeep V \\ ${ }^{1,6,7}$ Resident, P.G. Department of Radiodiagnosis, V.S.S. Institute of Medical Sciences and Research, Burla, \\ Sambalpur, Odisha, India \\ ${ }^{2,4,5}$ Associate Professor, P.G. Department of Radiodiagnosis, V.S.S. Institute of Medical Sciences and \\ Research, Burla, Sambalpur, Odisha, India \\ ${ }^{3}$ Professor and Head, P.G. Department of Radiodiagnosis, V.S.S. Institute of Medical Sciences and \\ Research, Burla, Sambalpur, Odisha, India \\ Corresponding Author \\ Dr Subhasish Panda \\ Resident, P.G. Department of Radiodiagnosis, \\ V.S.S. Institute of Medical Sciences and Research, Burla, Sambalpur, Odisha, India \\ Email: drsubhasishpanda15@gmail.com
}

\begin{abstract}
Superior mesenteric artery syndrome is a rare acquired disorder where the third part of duodenum is compromised between the superior mesenteric artery anteriorly and the aorta and lumbar spine posteriorly. We report an interesting case of a 17 year old asthenic female presenting with recurrent bouts of epigastric pain along with repeated episodes of vomiting. She was subjected to ultrasound examination of the abdomen and subsequent computerised tomography which revealed a dilated stomach, dilatation of first and second part of duodenum with compression of its third part between superior mesenteric artery and the aorta. A diagnosis of superior mesenteric artery syndrome was made. This was corroborated with surgical findings of extrinsic compression of third part of duodenum between superior mesenteric artery and the aorta.
\end{abstract}

Keywords- aortomesenteric angle, aortomesenteric distance, duodenum, superior mesenteric artery.

\section{INTRODUCTION}

Superior mesenteric artery syndrome (SMAS) is a rare but well recognised acquired disorder where the third part of duodenum is compromised between the superior mesenteric artery (SMA) contained in the root of the mesentery anteriorly and the aorta and lumbar spine posteriorly resulting in partial or complete acute duodenal obstruction or chronic intermittent obstruction ${ }^{[1]}$. Only around 400 cases of this condition have been reported thus far. It is potentially life-threatening and treated surgically in case of failure of conservative management ${ }^{[2]}$.

\section{CASE REPORT}

A 17 year old female patient, who was previously healthy, presented to the Surgery Out Patients Department of our Institution, with acute symptoms of colicky pain over upper abdomen and vomiting. She also mentioned experiencing similar episodes during the past three years. In addition, she reported weight loss over the last one year. She weighed 37 kilograms at presentation. Clinical examination revealed her vitals to be stable. Abdominal examination showed epigastric fullness without any tenderness on palpation. Her routine blood and urine 


\section{JMSCR Vol||04||Issue||11||Page 13692-13695||November}

examination reports did not reveal any abnormality. A clinical diagnosis of small bowel obstruction was made by the surgeon and referred to our department for further investigations.

Ultrasound examination revealed distension of the stomach and dilated duodenum upto its second part with abrupt distal tapering and apparent compression of the third part of duodenum between the anteriorly located superior mesenteric artery and the aorta posteriorly. Beyond this bowel segment, the small bowel loops were collapsed. Aortomesenteric distance was measured to be 5 to $6 \mathrm{~mm}$ (Fig 1).

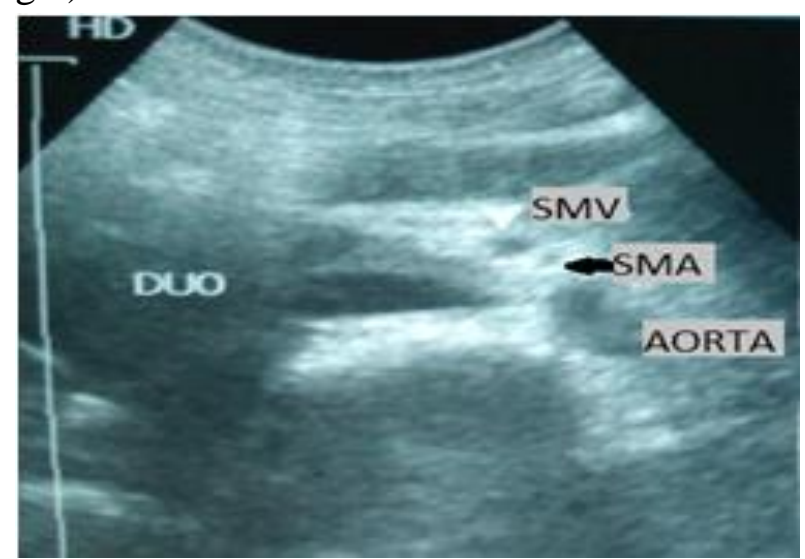

Fig 1. Ultrasound of the abdomen showing abrupt tapering of duodenal loop proximal to apparent compression between SMA and Aorta.

Computerised tomography reiterated the ultrasound findings with beaking of third part of duodenum proximal to spatial compromise between superior mesenteric artery and the aorta with nonvisualization of oral contrast distally. (Fig 2).

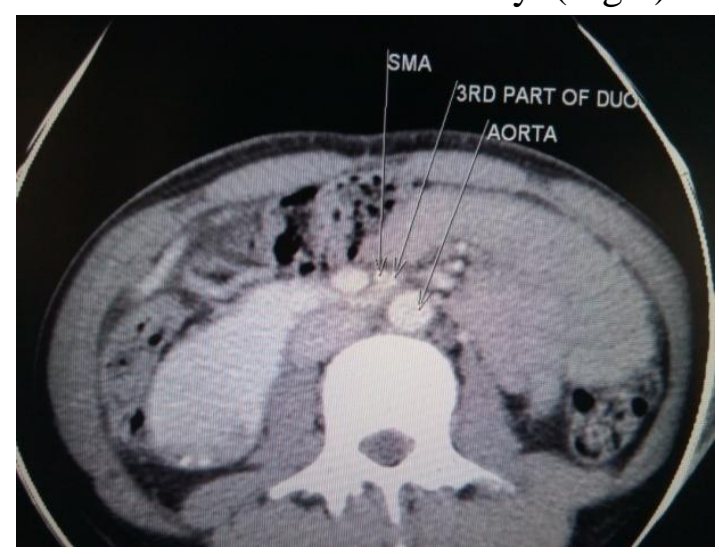

Fig 2. Axial Computerised Tomography (CT) of the abdomen showing narrowing of the $3^{\text {rd }}$ part of duodenum between SMA and Aorta; Aortomesenteric distance- $0.53 \mathrm{~cm}$.
On prone positioning, oral contrast was seen to pass beyond the site of previous obstruction (Fig 3).

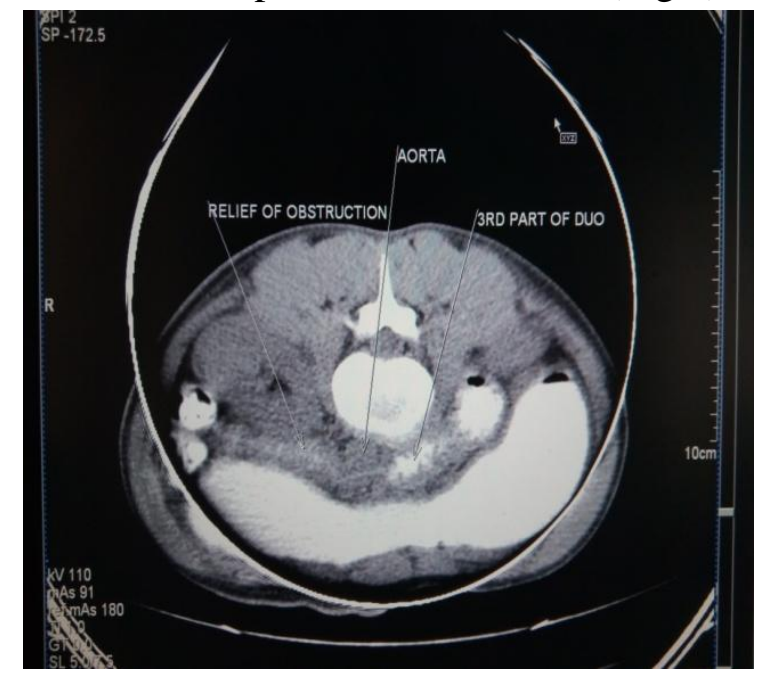

Fig 3. Prone CT scan showing passage of oral contrast beyond $3^{\text {rd }}$ part of duodenum

The aorto-mesenteric angle measured at the origin of superior mesenteric artery was 17 degrees and the aorto-mesenteric distance at the level of duodenum was around $5 \mathrm{~mm}$. (Fig 4)

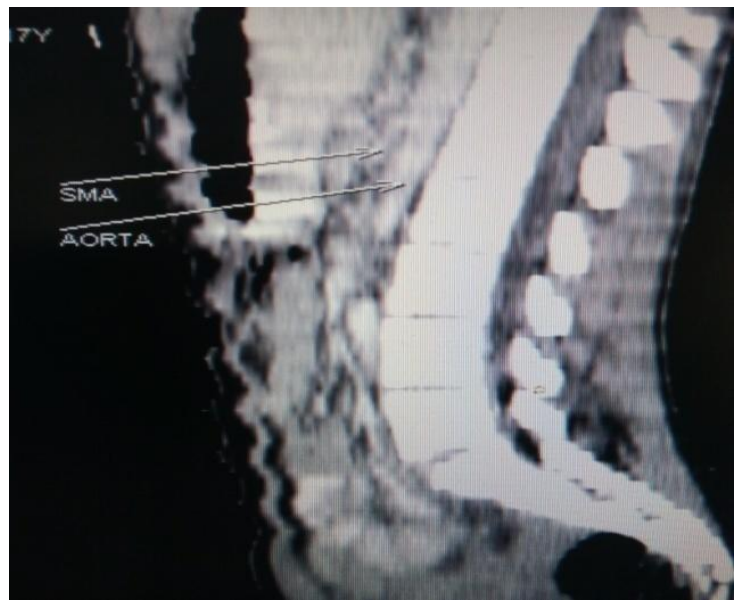

Fig 4. Sagittal CT image showing the hyper-acute aorto-mesenteric angle.

A provisional diagnosis of small bowel obstruction secondary to superior mesenteric artery syndrome was made. She underwent conservative management (nasogastric decompression, hyperalimentation, posturing manoeuvres) which failed to alleviate her symptoms. Thereafter, explorative laparotomy was undertaken which confirmed the radiological findings, and a duodeno-jejunostomy was done. The patient's condition significantly improved post-operatively and she was discharged. 


\section{DISCUSSION}

Superior mesenteric artery syndrome (SMAS) was first described by Rokitanski in $1861^{[3]}$. It occurs more commonly in female children and adolescents and in those who have thin body habitus because of paucity of duodenal fat pad ${ }^{[4]}$. The SMA is normally encased in fatty and lymphatic tissue at its origin which is important for maintaining a wide enough aorto-mesenteric angle and distance, thereby providing protection to the duodenum against compression between the SMA and the aorta ${ }^{[5]}$. A reduction in the amount of mesenteric or retroperitoneal fat appears contributory to Superior Mesenteric Artery Syndrome. Our patient mirrored these classic features.

SMAS can be due to a myriad of conditions such as an unusual low origin of the SMA, high insertion of the duodenum at the ligament of Treitz (acts as a suspensory ligament for the SMA), severe weight loss, an aortic aneurysm, retroperitoneal tumor, compression of the duodenum due to peritoneal adhesions, body plaster cast, scleroderma \& lupus erythematosus (reduced duodenal peristalsis) ${ }^{[4]}$.

Compression of duodenum by SMA characteristically produces gastrointestinal symptoms, including nausea, vomiting, abdominal pain and weight loss, which were seen in our patient ${ }^{[5]}$.

In our patient, ultrasound examination displayed a distended stomach and duodenum (upto its second part) with abrupt cut-off at the level of the third part of duodenum between the anteriorly located superior mesenteric artery and the aorta posteriorly. Beyond this bowel segment, the small bowel loops were collapsed.

At the level of the third part of the duodenum which lies between the SMA and the aorta, the distance between these two vessels (aorto-mesenteric distance) is $10-28 \mathrm{~mm}{ }^{[5]}$. However, aortomesenteric distance in our patient was measured to be only 5 to $6 \mathrm{~mm}$ presumably due to depleted periduodenal fat. Unal et al observed a reduction in the distance between the SMA and the aorta in SMAS cases in comparison to control subjects. Moreover, they found a high correlation between the aorto-mesenteric distance reduction at the level of duodenum and clinical symptoms of SMAS ${ }^{[6]}$.
Contrast Enhanced Computerised Tomography of the abdomen which is considered to provide the definitive diagnosis for this entity revealed beaking of third part of duodenum proximal to spatial compromise between superior mesenteric artery and the aorta with non-visualization of oral contrast distally ${ }^{[7]}$. Normally, the superior mesenteric artery (SMA) emerges from the anterior aspect of the abdominal aorta, making an acute angle with it at its origin (identified as the aorto-mesenteric angle) at the level of transpyloric plane (at lower border of L1 vertebral body). In normal individuals, this angle ranges between $38^{\circ}$ and $60^{\circ}$ with a mean of $45^{\circ[8]}$. But in our patient, the aorto-mesenteric angle was too acute measuring only 17 degrees with an aortomesenteric distance of around $5 \mathrm{~mm}$ measured at the level of duodenum as in ultrasound.

In some cases the obstruction is related to posture which can be released by turning the patient to a prone or left lateral decubitus position ${ }^{[1]}$. This classic feature was seen in our patient.

\section{CONCLUSIONS}

Hence, in our patient, the demographic profile, clinical presentation including that of significant weight loss over a period of 6 months, reduced aorto-mesenteric angle (17 degrees) along with a decreased aorto-mesenteric distance $(5-8 \mathrm{~mm})$, combined with proximal duodenal dilatation and distally collapsed bowel loops with release of obstruction on prone scanning helped clinch the diagnosis of SMA Syndrome.

\section{REFERENCES}

1. Chapman A H. The Stomach and Duodenum. In: Sutton D ed. Textbook of Radiology and Imaging. 7th ed. London (UK): Churchill Livingstone; 2003:575-613.

2. Naheda HJ, Al-Sanae A, Wafa A. Superior Mesenteric Artery Syndrome: An Uncommon Cause of Intestinal Obstruction; Report of Two Cases and Review of Literature. Kuwait Medical Journal, 38 (3) (2006), pp. 241-244.

3. Rokitanski CV, Braumuller and Siedel: 1861. Lehrbuch der Pathologische Anatomie; Vienna. 3rd edn. Vol. 3:p. 187. 
4. Basroum MK, et al. Patient with both Wilkie syndrome and nutcracker syndrome. Vasc Med. 2008;13(3):247-50.

5. Ahmed AR, Taylor I (1997) Superior mesenteric artery syndrome. Postgrad Med 73:776-778

6. Unal B, Aktas A, Kemal G etal (2005) Superior mesenteric artery syndrome: $C T$ and ultrasonography findings. Diagn Interv Radiol 11(2):90-95

7. Md. Tabrez Aziz, Md. Omar Tabrez. Superior Mesenteric Artery Syndrome: A Rare Case Report. International Journal of Science and Research 2016; 5 (5): 17-18.

8. Baltazar U, Dunn J, Floresguerra C etal (2000) Superior mesenteric artery syndrome: an uncommon cause of intestinal obstruction. South Med J 93(6):606-608 